\title{
TRAPPED MODES ANALYSIS FOR THE ELETTRA BOOSTER DCCT INSTALLATION
}

\author{
P. Craievich*, C. Bontoiu, G. Ciani and M. Ferianis, Sincrotrone Trieste, ELETTRA, Italy
}

\section{Abstract}

In the new Elettra full energy injector, bunch charge measurements will be performed by different types of current transformers (CT), depending on their position (single pass or multi pass sections). In the single pass sections (Linac and Transfer lines) a new type of current transformer (in-flange CT by Bergoz) will be used. Main advantage of this device is a compact and reliable design; they are also specially suited in space critical application. For the booster ring a standard DC current transformer will be used to measure the DC component of the circulating beam current. The housing has been developed in house, including the magnetic shield and the ceramic gap in the vacuum chamber. Furthermore, calculations of the trapped modes in the current monitor housing are described. Longitudinal coupling impedance and loss factors for these resonant modes are estimated and we showed that dissipated power is not critical with ELETTRA booster parameters.

\section{INTRODUCTION}

The Elettra new full energy injector is based on a 100 $\mathrm{MeV}$ linac pre-injector, a $2.5 \mathrm{GeV}$ booster synchrotron and two new beam transfer lines [1]. Table 1 lists some parameters of the booster ring that will be used in the next paragraph [2]. The average beam current is commonly measured by passing bunches through the center of a toroidal transformer. For the booster ring a standard DC current transformer (DCCT) will be used to measure the DC component of the circulating beam current. The DCCT is a parametric current transformer (PCT) by Bergoz Instrumentation [3]. It consists of three units: a toroidal sensor (made up of a number of toroidal cores, packed together and shielded with layers of high permeability materials), a front-end electronics box and a back-end power supply and control chassis. The operation of the DCCT is based on the different shape of the $B-H$ curve of a magnetic material with or without the presence of a DC current. The asymmetry of the $B-H$ curve allows the extraction of the DC component of the beam passing through the core. A modulator circuit is the combination of an excitation generator and the two cores, which have identical windings, but opposite orientation. The generator drives the two cores into saturation, a sense winding picks up the second harmonic of the resulting modulated signal and the demodulator processes it. A compensation circuit processes the output of the demodulator, and generates a current which forces the output signal back to zero: the DC current is measured by means of this compensation current.

*paolo.craievich@elettra.trieste.it

06 Instrumentation, Controls, Feedback \& Operational Aspects
The housing has been developed in house, including the magnetic shield and the ceramic gap in the vacuum chamber. Figure 1 shows a schematic drawing of the vacuum chamber for the housing of the DCCT that will be used in Elettra booster. The DCCT reads the sum of all currents passing through its toroidal core: that's why the electrical conductivity of the vacuum chamber must be interrupted appropriately. The adoption of the ceramic break introduces discontinuities in the vacuum chamber, as shown in the figure. In this paper calculations of the trapped modes in the current monitor housing are described. Longitudinal coupling impedance and loss factors for these resonant modes are estimated.

Table 1: Booster parameters list (SB: single bunch, MB: multi bunch).

\begin{tabular}{|l|c|c|c|l|}
\hline Parameter & Sy. & SB & MB & Unit \\
\hline Pulse charge & $Q_{p}$ & 0.16 & 2 & $n C$ \\
\hline Pulse length & $t_{p}$ & 2 & 100 & $n s$ \\
\hline Peak current & $I_{\text {peak }}$ & 80 & 20 & $m A$ \\
\hline Average current & $I_{a v}$ & 0.4 & 5 & $m A$ \\
\hline Injection rate & & 1.250 & 15.625 & $m A / s$ \\
\hline Number of bunches & $N_{b}$ & 1 & 50 & \\
\hline
\end{tabular}

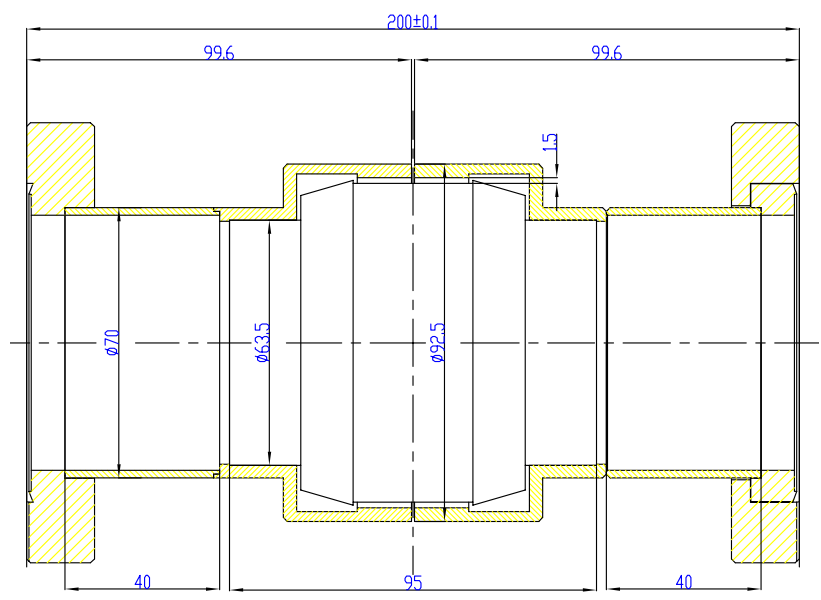

Figure 1: Schematic drawing of the vacuum chamber for the housing of DCCT with its main geometric dimensions.

\section{DISSIPATED POWER IN THE DCCT}

Two operational mode of the ELETTRA booster are foreseen: single bunch (SB) and multi bunch (MB) as indicated in the Table 1 . The SB mode foresees an average current of $0.4 m A$ and a bunch charge of $0.16 n C$ while the MB

T03 Beam Diagnostics and Instrumentation 
mode consists in an average current of $5 \mathrm{~mA}$ and a bunch charge of $0.04 n C$. A RMS single bunch length $\sigma_{t}=20 p s$ is assumed. The average dissipated power by an uncorrelated train of bunches is given by:

$$
P_{d}=q_{b} k_{L} I_{a v}
$$

where $q_{b}$ is the single bunch charge, $I_{a v}$ is the average beam current and $k_{L}$ the loss factor. The term $q_{b} I_{a v}$ is $6.4 \cdot 10^{-2} n C m A$ and $20 \cdot 10^{-2} n C m A$ for $\mathrm{SB}$ and MB respectively. The MB operational mode is taken here in consideration since it is the worse case.

The loss factor for a resonator with $\sigma_{t} \ll 1 / \omega_{R}$ is:

$$
k_{L}=\frac{\omega_{R}}{2} \frac{R}{Q}
$$

where $\omega_{R}$ is the resonant frequency and $R / Q$ is the geometric parameter of the trapped modes in the DCCT housing. In addition the loss factor $k_{L}$ can be calculated by means of the longitudinal coupling impedance $Z(\omega)$, as in the following formula:

$$
\frac{1}{\pi} \int_{0}^{\infty} \Re Z(\omega) d \omega \cong k_{L}
$$

The coupling impedance of an accelerator component can be calculated by coaxial wire methods applying the following relation $[4,5]$ :

$$
Z(\omega)=2 R_{0} \frac{s_{21}^{r e f}(\omega)-s_{21}^{\text {dut }}(\omega)}{s_{21}^{\text {dut }}(\omega)}
$$

where $s_{21}^{d u t}$ is the transmission parameter of the resonant cavity under test, $s_{21}^{r e f}$ is the transmission parameter of the unperturbed coaxial line of the same length and $R_{0}$ is the characteristic impedance of the coaxial line. Considering an inner conductor of radius $0.1 \mathrm{~mm}$ the characteristic impedance is equivalent to $344 \Omega$. In this paper the transmission parameters in the formula (4) are calculated by means of HFSS code [6] and applying the formula (3) the loss factor is calculated. Next section reports the comparison between the results obtained using formulas (2) and (3). The electromagnetic simulations have pointed out the presence of two resonant modes: the $T M_{010}$ - like mode in the DCCT housing and the TEM - like mode between the outer surface and the support of the ceramic gap.

Figure 2 shows the plots of the real and imaginary parts of the longitudinal coupling impedances while in the table 2 the resonant frequencies, Q-factors, loss factors and dissipated power for the two trapped modes are listed. One can observe that the dissipated power is negligible considering the worse case for the booster ring parameters. Stainless steel is used as material of the DCCT housing and here the conductivity is equivalent to $1.1 \cdot 10^{6} \mathrm{~S} / \mathrm{m}$. Alumina is the material of the ceramic gap with relative dielectric constant $\epsilon_{r}=9.8$.

Figures 3 and 4 show the electric and magnetic field distributions for the $T M_{010}$ - like and TEM - like resonant 06 Instrumentation, Controls, Feedback \& Operational Aspects
Table 2: Parameters of the trapped modes in the DCCT.

\begin{tabular}{|c|c|c|c|c|}
\hline Mode & $f_{r}$ & $\mathrm{Q}$ & $k_{\text {loss }}$ & $P_{d}$ \\
\hline & {$[G H z]$} & & {$[V / n C]$} & {$[W]$} \\
\hline$T M_{010}-$ like & 2.6175 & 1007 & 78.4 & 0.02 \\
\hline$T E M-$ like & 1.0150 & 199 & 26.5 & 0.01 \\
\hline
\end{tabular}
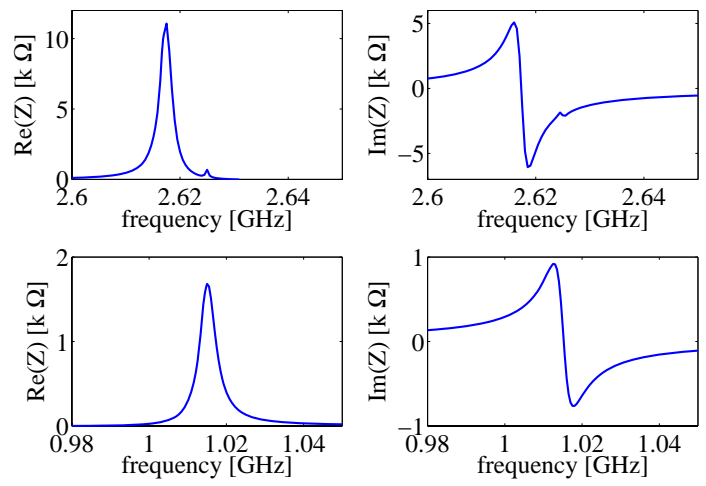

Figure 2: Longitudinal coupling impedances for the $T M_{010}$ - like mode (upper) and TEM - like mode (bottom).

modes, respectively. From figure 4 we can note that magnetic field of the TEM - like mode enter into the small gap but nevertheless the dissipated power is negligible.
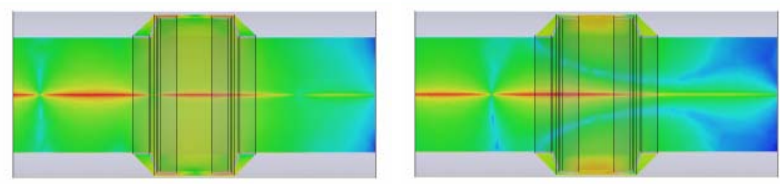

Figure 3: Electric (left) and magnetic (right) field distribution of the $T M_{010}$ - like resonant mode in the vacuum chamber.
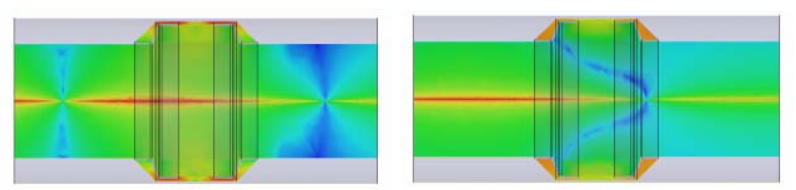

Figure 4: Electric (left) and magnetic (right) field distribution of the $T E M-$ like resonant mode in the vacuum chamber.

\section{LOSS FACTOR CALCULATIONS}

As seen in the previous section the loss factor for a resonant mode can be directly calculated with the formula (2) or indirectly calculated with the formula (3). In this section the loss factors obtained by two different approaches are compared. For this scope the geometry of the DCCT housing was simplified using a pill box with radius $41.24 \mathrm{~mm}$, 
gap filled with ceramic material of length $37 \mathrm{~mm}$ and beam pipe with radius $31 \mathrm{~mm}$. The $R / Q$, the resonant frequency and the Q-factor were calculated by HFSS eigensolver and the results obtained with the two methods are reported in table 3. Q-factor can be calculated as the ratio between resonant frequency and bandwidth at $-3 \mathrm{~dB}$ of the real part of the impedance. The relative error between the two methods is approximately $9 \%$ and $16 \%$ for loss factor and Q-factor, respectively.

Table 3: Comparison between eigensolver and coaxial wire method results for the $T M_{010}-$ like mode.

\begin{tabular}{|c|c|c|c|}
\hline \multicolumn{4}{|c|}{ Eigensolver } \\
\hline$f_{r}$ & $R / Q$ & $Q$ & $k_{\text {loss }}$ \\
\hline$[G H z]$ & {$[\Omega]$} & & {$[V / n C]$} \\
\hline 2.175 & 18.4 & 992 & 126 \\
\hline \multicolumn{4}{|c|}{ Wire method } \\
\hline$f_{r}$ & & $Q$ & $k_{\text {loss }}$ \\
\hline$[G H z]$ & & {$[V / n C]$} & {$[V / n C]$} \\
\hline 2.239 & & 829 & 137 \\
\hline
\end{tabular}

\section{CONCLUSION}

This note addresses the analysis of the trapped modes in the current monitor housing that will be used in the Elettra booster ring. The analysis has picked out two trapped resonant modes: the $T M_{010}$ - like and TEM - like, respectively. The longitudinal coupling impedances was calculated using the wire methods and the loss factor was obtained integrating the real part of the impedance. Results showed that the dissipated power from both trapped modes is negligible applying the nominal average current and bunch charge for the circulating multi bunch beam in the ELETTRA booster ring.

\section{Acknowledgments}

Paolo Craievich would like to thank Cristina Pasotti for helpful suggestions about Ansoft HFSS code and Simone Di Mitri for interesting discussion about booster parameters list.

\section{REFERENCES}

[1] M. Svandrlik et al., "ELETTRA new full energy injector status report”, EPAC'06, June 2006, Edinburgh, Scotland.

[2] F. Iazzourene, Booster Review Meeting, April 2005.

[3] Bergoz Instrumentation, http://www.bergoz.com.

[4] H. Hahn and F. Pedersen, "On coaxial wire measurements of the longitudinal coupling impedance", BNL 50870, UC-28, April 1978.

[5] A. Argan, L. Palumbo, M.R. Masullo and V.G. Vaccaro, "On the Sands and Rees measurement method of

06 Instrumentation, Controls, Feedback \& Operational Aspects 